\title{
UPLC-QTOFMS-based metabolomic analysis of the serum of hypoxic preconditioning mice
}

\author{
JIE LIU ${ }^{1-3}$, GANG ZHANG ${ }^{2-4}$, DEWEI CHEN ${ }^{2,3,5}$, JIAN CHEN $^{2,3,5}$, ZHI-BIN YUAN $^{1-3}$, ER-LONG ZHANG ${ }^{1-3}$, \\ YI-XING GAO ${ }^{1-3}$, GANG XU ${ }^{1-3}$, BING-DA SUN ${ }^{1-3}$, WENTING LIAO ${ }^{6,7}$ and YU-QI GAO ${ }^{1-3}$ \\ ${ }^{1}$ Institute of Medicine and Hygienic Equipment for High Altitude Region, College of High Altitude Military Medicine; \\ ${ }^{2}$ Key Laboratory of High Altitude Medicine, Ministry of Education; ${ }^{3}$ The Key Laboratory of High Altitude Medicine, \\ People's Liberation Army; Departments of ${ }^{4}$ High Altitude Military Hygiene, and ${ }^{5}$ Pathophysiology and High Altitude \\ Physiology, College of High Altitude Military Medicine, Third Military Medical University, Chongqing 400038; \\ ${ }^{6}$ Department of Pharmaceutical Analysis, School of Pharmacy; ${ }^{7}$ Key Laboratory of Drug Quality Control and \\ Pharmacovigilance, Ministry of Education, China Pharmaceutical University, Nanjing, Jiangsu 210009, P.R. China
}

Received March 15, 2017; Accepted July 24, 2017

DOI: $10.3892 / \mathrm{mmr} .2017 .7493$

\begin{abstract}
Hypoxic preconditioning (HPC) is well-known to exert a protective effect against hypoxic injury; however, the underlying molecular mechanism remains unclear. The present study utilized a serum metabolomics approach to detect the alterations associated with HPC. In the present study, an animal model of HPC was established by exposing adult BALB/c mice to acute repetitive hypoxia four times. The serum samples were collected by orbital blood sampling. Metabolite profiling was performed using ultra-performance liquid chromatography-quadrupole time-of-flight mass spectrometry (UPLC-QTOFMS), in conjunction with univariate and multivariate statistical analyses. The results of the present study confirmed that the HPC mouse model was established and refined, suggesting significant differences between the control and HPC groups at the molecular levels. HPC caused significant metabolic alterations, as represented by the significant upregulation of valine, methionine, tyrosine, isoleucine, phenylalanine, lysophosphatidylcholine (LysoPC; 16:1), LysoPC (22:6), linoelaidylcarnitine, palmitoylcarnitine, octadecenoylcarnitine, taurine, arachidonic acid, linoleic acid,
\end{abstract}

Correspondence to: Dr Wenting Liao, Department of Pharmaceutical Analysis, School of Pharmacy, China Pharmaceutical University, 24 Tong Jia Xiang, Nanjing, Jiangsu 210009, P.R. China E-mail: lwting84@163.com

Professor Yu-Qi Gao, Institute of Medicine and Hygienic Equipment for High Altitude Region, College of High Altitude Military Medicine, Third Military Medical University, 30 Gao Tan Yan Street, Chongqing 400038, P.R. China

E-mail: gaoy66@yahoo.com

Key words: hypoxic preconditioning, metabolomics, ultraperformance liquid chromatography-quadrupole time-of-flight mass spectrometry, metabolic pathway oleic acid and palmitic acid, and the downregulation of acetylcarnitine, malate, citrate and succinate. Using MetaboAnalyst 3.0, a number of key metabolic pathways were observed to be acutely perturbed, including valine, leucine and isoleucine biosynthesis, in addition to taurine, hypotaurine, phenylalanine, linoleic acid and arachidonic acid metabolism. The results of the present study provided novel insights into the mechanisms involved in the acclimatization of organisms to hypoxia, and demonstrated the protective mechanism of HPC.

\section{Introduction}

Hypoxia appears in a number of extreme environments, including high altitudes, the deep sea, and during aviation, and occurs in numerous diseases, including cardiovascular and respiratory failures, neurological disorders and cancer. Hypoxic preconditioning (HPC), motivated by the repetitive exposure of organisms, organs, tissues and cells to hypoxia is an intrinsic cytoprotective strategy, existing widely in the heart, brain, kidney, liver, intestine and other organs (1).

Previously, numerous studies have indicated that HPC may protect an organism against hypoxic injury (2-5). Additionally, the acute repetitive HPC mouse model has been widely utilized to study animal behavior, endogenous metabolites, metabolic pathways and intrinsic protective mechanisms under hypoxic conditions (6,7). A previous study demonstrated that the brain homogenates from preconditioned mice were able to strengthen the tolerance to hypoxia and protect the animals from hypoxic injury (8). Recent studies have examined the potential biomarkers of HPC $(9,10)$. Cui et al (11) performed a proteomic study to profile the patterns of protein expression in HPC mouse brains. Although the protective effect of HPC is known, the underlying mechanisms remain unclear, particularly at the endogenous metabolite level.

Metabolomics is a top-down systemic biological approach, whereby metabolic responses to physiological interventions or environmental factors are analyzed and modeled (12). Therefore, metabolomics represents an excellent developing 
prospect for capturing disease-specific metabolic signatures as putative biomarkers (13). Metabolomics appears to be a promising approach to identifying metabolite-based biomarkers and revealing the underlying mechanism of neurodegenerative diseases (14), cardiovascular disease (15), and cancer (16). Recently, a study identified the molecular alterations associated with HPC mouse brains using an ultra-high performance liquid chromatography-coupled high resolution mass spectrometry-based metabolomics approach (17). This strategy exemplified the ability of metabolomics to identify endogenous biomarkers and elucidate the protective mechanism of HPC.

In the present study, an acute repetitive HPC mouse model was established, and the serum metabolites were profiled using ultra-performance liquid chromatography-quadrupole time-of-flight mass spectrometry (UPLC-QTOFMS), in conjunction with univariate and multivariate data analyses. One of the purposes was to identify the differential serum metabolites in HPC associated with acute hypoxia and in normoxia controls. A further goal was to elucidate the mechanisms through which organisms acclimatize to hypoxia, in addition to the potential protective mechanism of HPC. The present study revealed the important metabolites and metabolic pathways in HPC and provided novel insights into the protective mechanism of HPC.

\section{Materials and methods}

Chemicals and reagents. Formic acid was obtained from Fluka (Sigma-Aldrich; Merck KGaA, Darmstadt, Germany). Acetone, ammonium formate, and citrate were purchased from Sigma-Aldrich (Merck KGaA). Methanol and acetonitrile (ACN) were chromatography grade (Merck KGaA). Valine, phenylalanine, methionine, uric acid, arachidonic acid, oleic acid, linoleic acid, palmitic acid and sodium succinate were obtained from Shanghai Jingchun Reagent Co., Ltd. (Shanghai, China). Ultrapure water was prepared using a Milli-Q water purification system (EMD Millipore, Billerica, MA, USA).

Animals and sample collection. Male BALB/c mice of 6-8 weeks old, weighing 18-22 g, were obtained from the Experimental Animal Center of the Third Military Medical University (Chongqing, China). A total of 30 BALB/C mice were randomly divided into the normoxic control $(\mathrm{H} 0)$, acute hypoxic (H1) and acute repetitive hypoxia for four times (HPC) groups. Mice were housed at $22 \pm 2^{\circ} \mathrm{C}$ and $60 \pm 10 \%$ relative humidity in a specific pathogen-free environment, with a 12-h light/dark cycle and ad libitum access to food and water.

The animal model of HPC was established according to a method described previously (18). A weighed mouse was placed in a $125-\mathrm{ml}$ jar, which was sealed airtight with a rubber plug. The mouse was taken out of the jar immediately following the appearance of the first asthmoid respiration (a sign of the hypoxia tolerance limit); this was the first instance of hypoxia exposure. Subsequently, the mouse was moved to a new, similar airtight jar in order to duplicate a progressive hypoxic environment three more times; the time of hypoxia tolerance in each mouse (from the beginning of the first airtight exposure to the final asthmoid respiration) was recorded. The $\mathrm{H} 1$ group was subjected to hypoxia only 1 time, and the H0 group did not undergo the hypoxic treatment. According to the following formula, the standard tolerance time was computed: $\mathrm{T}=\mathrm{t} /(\mathrm{v}-\mathrm{w}) / 0.94 \times 100$ ( $\mathrm{T}$, standard tolerance time; $\mathrm{t}$, hypoxia tolerance time; $v$, jar volume; w, mouse weight).

At the end of the experiment, the animals were anesthetized and blood samples withdrawn via orbital puncture. Subsequently, the samples were maintained at room temperature for $30 \mathrm{~min}$, followed by centrifugation at $4^{\circ} \mathrm{C}$ and $1,500 \mathrm{x} \mathrm{g}$ for $10 \mathrm{~min}$, and preserved at $-80^{\circ} \mathrm{C}$. All animal studies were approved by the Third Military Medical University Animal Care and Use Committee.

Serum sample preparation. The serum samples were prepared according to a previous report (19). A 100- $\mu$ l aliquot of serum sample was mixed with $10 \mu \mathrm{l}$ L-2-chlorophenylalanine ( $1 \mathrm{mg} / \mathrm{ml}$ in $\mathrm{H}_{2} \mathrm{O}$ ), followed by the addition of $400 \mu \mathrm{l}$ methanol/acetonitrile/acetone (1:1:1, v/v/v). Following vigorous agitation for $1 \mathrm{~min}$ and incubation on ice for $10 \mathrm{~min}$, the mixture was centrifuged at $13,000 \mathrm{x}$ g for $15 \mathrm{~min}$ at $4^{\circ} \mathrm{C}$ to precipitate the protein. The supernatant was filtered through a $0.22-\mu \mathrm{m}$ syringe filter and transferred into the sampling vial for UPLC-QTOFMS analysis. The serum samples from each group were alternated in a random order for sequential analysis in order to avoid technical errors originating from sample preparation and analysis.

UPLC-QTOFMS analysis. UPLC-QTOFMS analysis was performed on an Agilent 1290 Infinity LC system coupled to Agilent 6530 Accurate-Mass Quadrupole Time-of-Flight (Q-TOF) mass spectrometer (Agilent Technologies, Inc., Santa Clara, CA, USA). The chromatographic separations were performed on an ACQUITY UHPLC HSS T3 C18 column (2.1x100 mm; $1.8 \mu \mathrm{m}$; Waters Corporation, Milford, MA, USA) maintained at $45^{\circ} \mathrm{C}$. The flow rate was $400 \mu \mathrm{l} / \mathrm{min}$, and the injection volume was $4 \mu \mathrm{l}$. The mobile phase consisted of $0.1 \%$ formic acid (A) and ACN modified with $0.1 \%$ formic acid (B). A linear gradient was applied as follows: $2 \%$ B at 0-3 min; 2-95\% B at 3-20 min; and 95\% B at 20-22 min, followed by a re-equilibration step of $5 \mathrm{~min}$.

An electrospray ionization source interface was set in positive and negative modes in order to monitor the maximum number of ions. The optimized conditions were as follows: Capillary voltage, $3.5 \mathrm{kV}$; drying gas flow, $11 \mathrm{l} / \mathrm{min}$; gas temperature, $350^{\circ} \mathrm{C}$; nebulizer pressure, $45 \mathrm{psi}$; fragmentor voltage, $120 \mathrm{~V}$; and skimmer voltage, $60 \mathrm{~V}$. Data were collected in the centroid mode from $100-1,100 \mathrm{~m} / \mathrm{z}$. The potential biomarkers were analyzed by tandem MS (MS/MS) in the Q-TOF. Nitrogen was used as the collision gas. The MS/MS analysis was performed on the mass spectrometer adjusted to different collision energies from 10-20 eV, according to the stability of each metabolite. The MS spectra were collected at $2 \mathrm{spectra} / \mathrm{s}$, and the MS/MS spectra were collected at $0.5 \mathrm{spectra} / \mathrm{s}$, with a medium isolation window $(\sim 4 \mathrm{~m} / \mathrm{z})$. The MS parameters set in the negative mode were similar to those in the positive mode.

Data handling. The data were preprocessed according to a method used previously, with minor modifications (19). The raw data in an instrument-specific format (.d) were converted to $\mathrm{mz}$ data formats using Agilent MassHunter Qualitative 
software (version B.02.00; Agilent Technologies, Inc.). The XCMS (version 1.40.0; metlin.scripps.edu/download) program was employed for nonlinear alignment of the data in the time domain, in addition to the automatic integration and extraction of the peak intensities. The XCMS parameters applied were the default settings except for the following: Full width at half maximum $(\mathrm{fwhm})=10$, bandwidth $(\mathrm{bw})=10$, and signal/noise ratio threshold (snthresh) $=5$. The variables that were notpresent in a minimum of $80 \%$ of the groups were filtered. An internal standard (L-2 chlorophenylalanine) was used for data quality control and normalization. In addition, the ion peaks generated by the internal standard were removed. The resulting three-dimensional matrix, including retention time, $\mathrm{m} / \mathrm{z}$ pairs, sample names and normalized ion intensities, was introduced into the multivariate data analysis.

The two datasets resulting from UPLC-QTOFMS in positive ion mode (ESI+) and negative ion mode (ESI-) were analyzed and validated using a multivariate statistical method. Each normalized dataset was imported into the SIMCA-P 13.0 software package (Umetrics, Umeå, Sweden) for principal component analysis (PCA) and partial least squares-discriminant analysis (PLS-DA), following mean-centering and pareto-scaling, the significantly different expression of peaks (variable importance; VIP $>1$ ) was identified by the software. The quality of the models was evaluated with the relevant R2 and Q2 discussed elsewhere (20). Statistically significant differences for the variables between the H0, H1, and HPC groups were tested using one-way analysis of variance and the Tukey post hoc test. The metabolites obtained from the ESI+ and ESI- modes of the UPLC-QTOFMS analysis were similar to a previous study (21). The quasi-molecular ion peak was identified to be similar to the accurate mass and retention time in the extracted ion chromatogram (EIC), and the most probable molecular formula was calculated using the Agilent MassHunter software. The commercial standard MS/MS spectrum was used to confirm the identified compound. Pathway analysis and visualization using the Kyoto Encyclopedia of Genes and Genomes (KEGG; www .genome.jp/kegg) pathway database was conducted using MetaboAnalyst $3.0(22,23)$.

\section{Results}

Animal model of HPC. The duration of tolerance of mice in the H1 and HPC groups was $17.2 \pm 3.3$ and $91.4 \pm 11.5 \mathrm{~min}$, respectively. The hypoxia tolerance of mice was prolonged with the increase in the time of hypoxia exposure, which suggested that the hypoxia preconditioned mouse model was successfully established.

Serum metabolic profiling by UPLC-QTOFMS. The coefficient of variation $(\mathrm{CV})$ of the internal standard was calculated. The results demonstrated that the $\mathrm{CV}$ was $<15 \%$, which demonstrated the robustness of the method. Therefore, differences observed between groups via multivariate statistical analysis were more likely to reflect varied metabolite profiles rather than analytical variation.

The typical total ion current chromatograms in positive ion mode (ESI+) and negative ion mode (ESI-) of UPLC-QTOFMS are presented in Fig. 1. A total of 1,008 and 922 peaks were

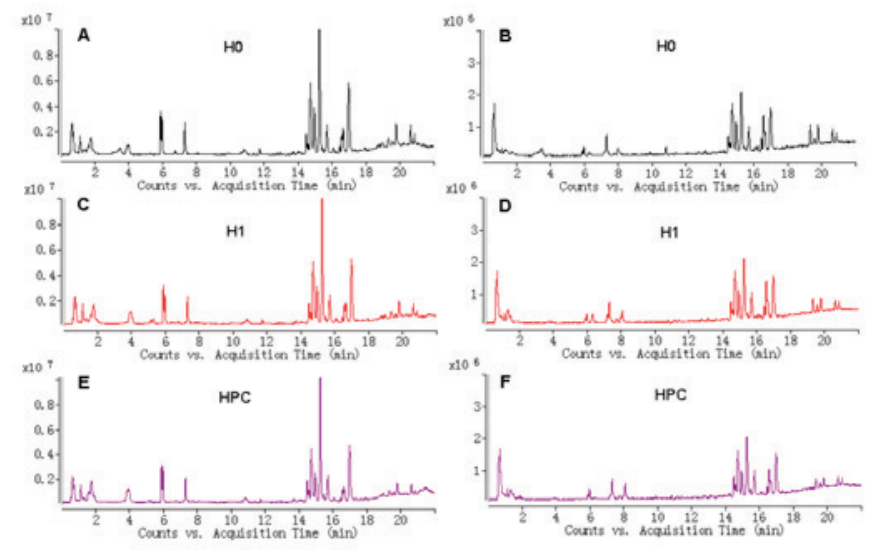

Figure 1. Typical total ion current chromatograms from (A) H0, (C) H1 and (E) HPC serum samples separated on LC ESI+, and (B) H0, (D) H1 and (F) HPC serum samples separated on LC ESI-. H0, normoxic control; H1, acute hypoxic; HPC, acute repetitive hypoxia; LC ESI+, liquid chromatography positive ion mode; LC ESI-, liquid chromatography negative ion mode.

obtained from UPLC-QTOFMS ESI+ (expressed as ESI+ dataset) and ESI- mode (expressed as ESI- dataset), respectively.

Multivariate statistical analysis. Following data normalization, the PCA was conducted on the dataset, which revealed a trend of intergroup separation on the scores plots. The plots of the PCA and PLS-DA scores obtained from the three groups were constructed based on the spectral data of the UPLC-QTOFMS ESI+ mode (Fig. 2) and UPLC-QTOFMS ESI- mode (Fig. 3). The two PLS-DA score plots demonstrated that the H0, H1 and HPC groups were able to be separated distinctly. The PLS-DA models were validated by a permutation test (99 times). As presented in Figs. 2C and 3C, the $\mathrm{R}^{2}$ intercept values of all the models and their $\mathrm{Q}^{2}$ intercept values correlated with the extent of overfitting were small, indicating satisfactory establishment of the models.

Identification of differential serum metabolites in HPC. In order to select the potentially different metabolites optimal for preferential study, metabolites which differed significantly among the groups following one-way ANOVA $(\mathrm{P}<0.05)$ were identified. Variables that significantly contributed towards clustering and discrimination were identified, according to the threshold values of variable importance in the projection (VIP $>1$ ), which were generated following PLS-DA processing. According to the above two different statistical methods, 25 significantly-altered metabolites were identified in HPC (Table I). The metabolites obtained from the positive and negative ion modes of the UPLC-QTOFMS analysis were similar to a previous study (19).

Metabolic pathway analysis. Metabolite profiling focuses on the analysis of a group of metabolites encompassed in a specific physiological pathway. A detailed analysis of the relevant pathways and networks of HPC was performed using MetaboAnalyst 3.0 (Table II). Consequently, the potential target metabolic pathway analysis (impact-value $\geq 0.10$ ) with MetaboAnalyst revealed that phenylalanine, tyrosine and tryptophan biosynthesis, valine, leucine and isoleucine 

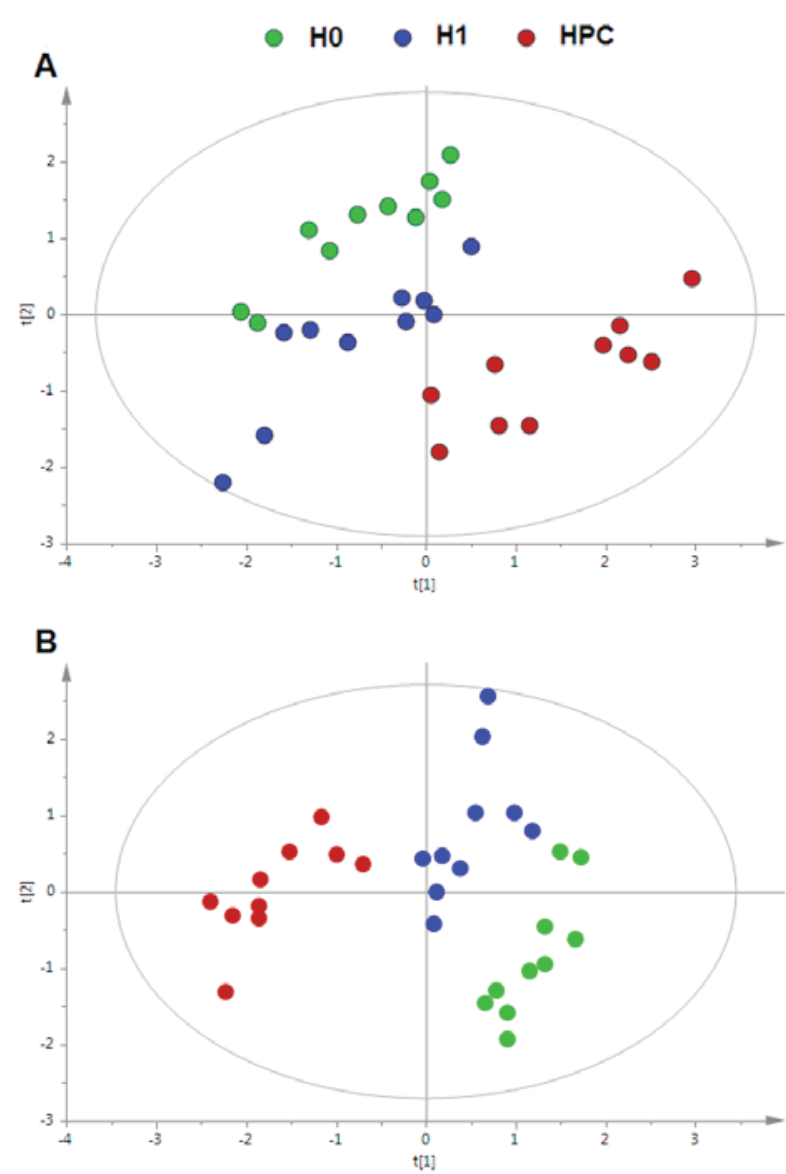

$R 2 X=0.695, R 2 Y=0.667, Q 2=0.595$

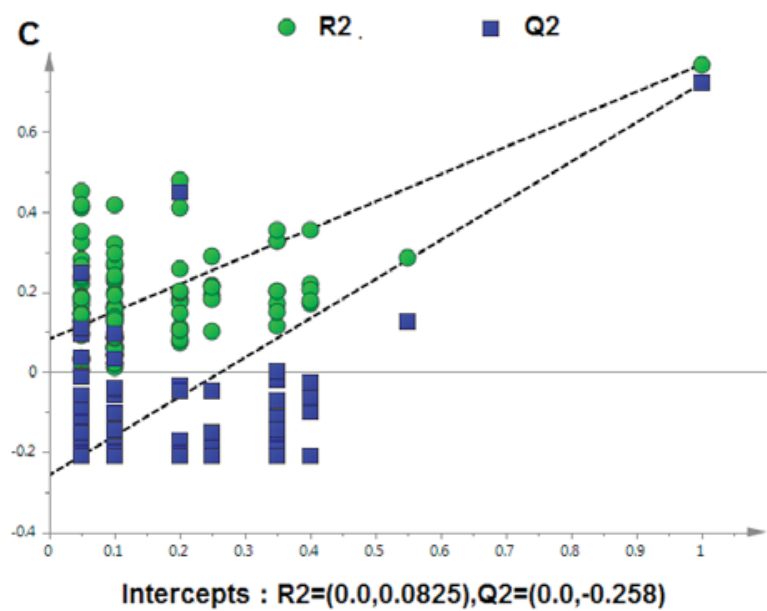

Figure 2. Multivariate data analysis based on serum spectral data of UPLC-QTOFMS positive ion mode. (A) Principal component analysis score map derived from UPLC-QTOFMS spectra containing H0, H1 and HPC groups. (B) Partial least squares-discriminant analysis score map derived from UPLC-QTOFMS spectra containing H0, H1 and HPC groups. (C) Validation plot obtained from 99 permutation tests. UPLC-QTOFMS, ultra-performance liquid chromatography-quadrupole time-of-flight mass spectrometry; H0, normoxic control; H1, acute hypoxic; HPC, acute repetitive hypoxia.

biosynthesis, taurine and hypotaurine metabolism, phenylalanine metabolism, linoleic acid metabolism, arachidonic acid metabolism, glyoxylate and dicarboxylate metabolism, and tyrosine metabolism were disrupted in HPC (Fig. 4). In
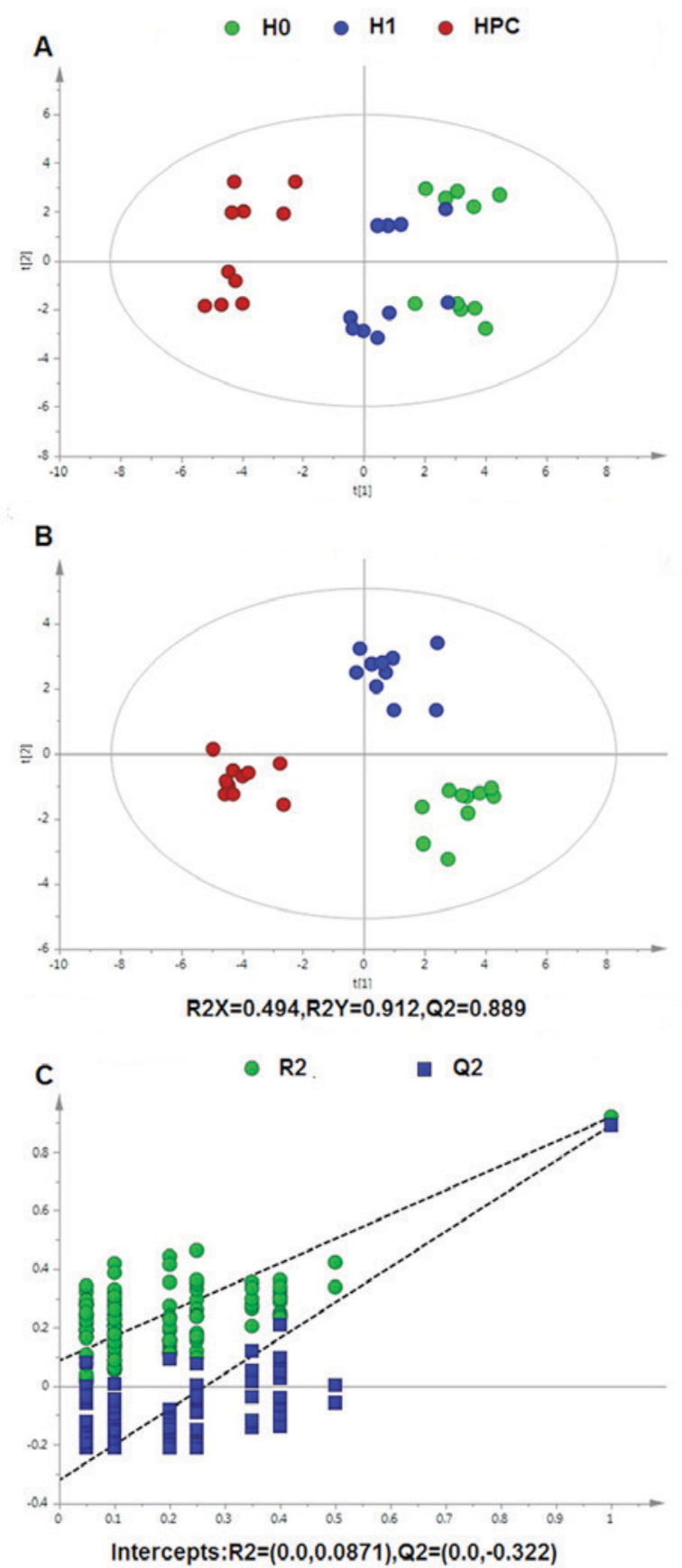

Figure 3. Multivariate data analysis based on serum spectral data of UPLC-QTOFMS negative ion mode. (A) Principal component analysis score map derived from UPLC-QTOFMS spectra containing H0, H1 and HPC groups. (B) Partial least squares-discriminant analysis score map derived from UPLC-QTOFMS spectra containing H0, H1 and HPC groups. (C) Validation plot obtained from 99 permutation tests. UPLC-QTOFMS, ultra-performance liquid chromatography-quadrupole time-of-flight mass spectrometry; H0, normoxic control; H1, acute hypoxic; HPC, acute repetitive hypoxia.

addition, further correlated pathways were constructed using the reference map obtained from the KEGG (Fig. 5).

\section{Discussion}

Hypoxic preconditioning has been widely accepted to be an endogenous protective effect; however, the underlying 


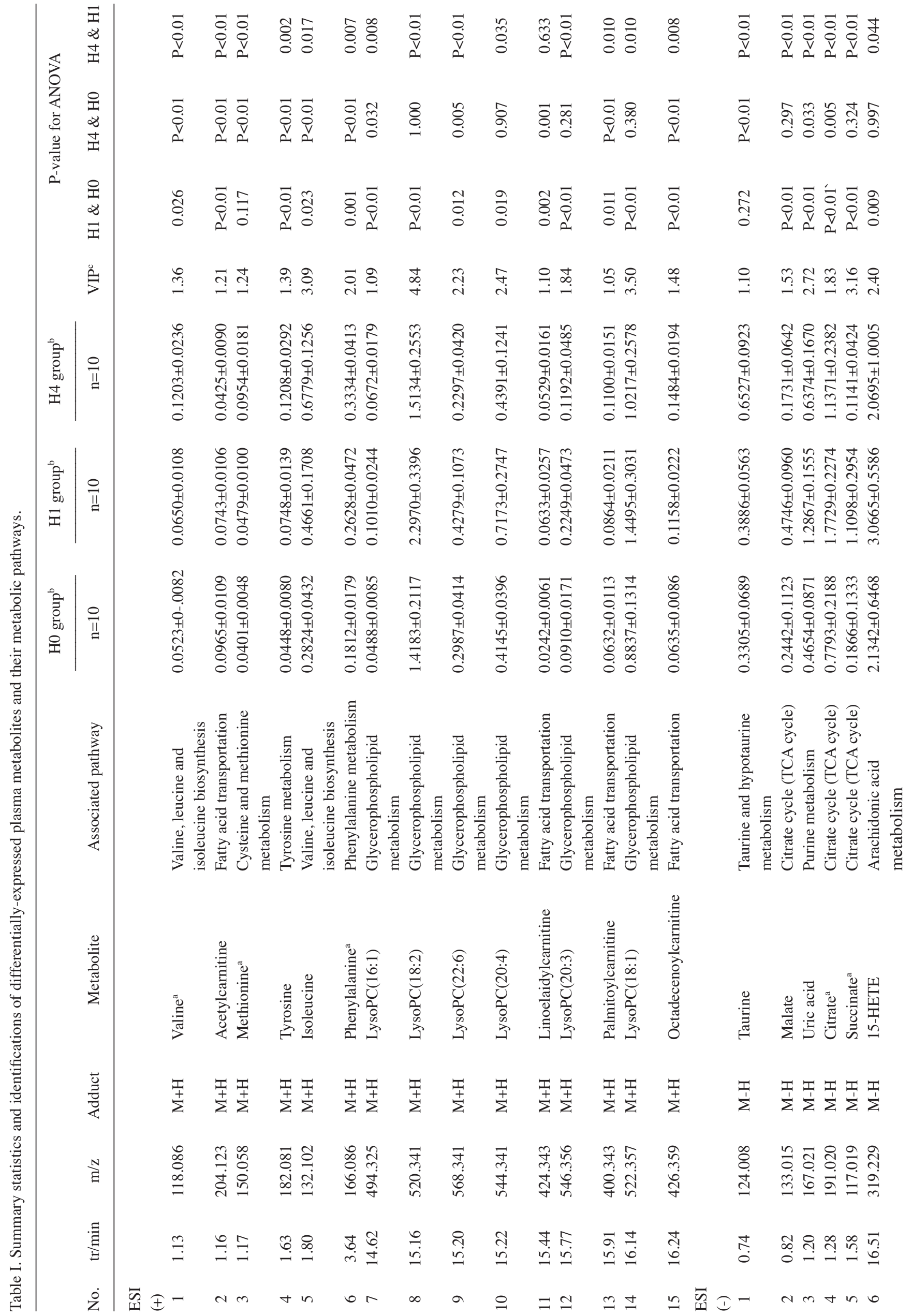




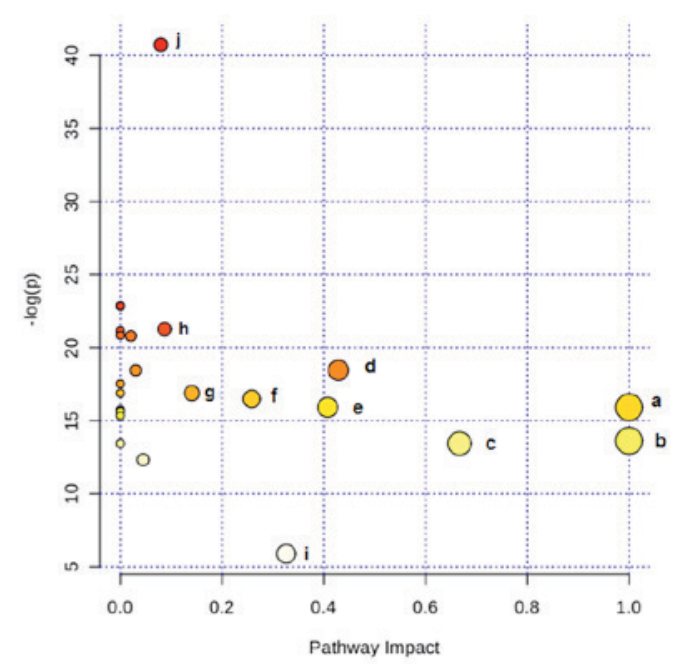

Figure 4. Altered metabolic pathways under hypoxia preconditioning. a, phenylalanine, tyrosine, and tryptophan biosynthesis; b, linoleic acid metabolism; c, valine, leucine, and isoleucine biosynthesis; $\mathrm{d}$, taurine and hypotaurine metabolism; e, phenylalanine metabolism; f, arachidonic acid metabolism; g, glyoxylate and dicarboxylate metabolism; h, tyrosine metabolism; i, citrate cycle.

mechanism has not yet been completely elucidated. In the present study, endogenous metabolites in the serum of HPC mice were analyzed by UPLC-QTOFMS. A total of 25 differentially-expressed metabolites were identified, including phenylalanine, tyrosine and tryptophan biosynthesis, valine, leucine and isoleucine biosynthesis, taurine and hypotaurine metabolism, phenylalanine metabolism, linoleic acid metabolism, arachidonic acid metabolism, and glyoxylate and dicarboxylate metabolism. The major metabolic patterns and plausible pathways in HPC mice are discussed below.

The results of the present study demonstrated that valine and isoleucine, the two branched-chain amino acids (BCAAs), were significantly increased in the H1 and HPC groups, with the highest levels in the HPC group. BCAAs are the main components of muscle protein and are important substrates for the production of ATP in muscle tissues. In hypoxic conditions, due to the lack of oxygen, mitochondrial dysfunction leads to a restrictive utilization of BCAAs; the two aromatic amino acids, phenylalanine and tyrosine, were significantly increased in the H1 and HPC groups, with the highest levels in the HPC group. Aromatic amino acids are metabolized primarily in the liver. The continued increase may be associated with the decreased metabolic capacity of the liver in hypoxic environments $(24,25)$. These alterations indicated that amino acids may selectively decrease the metabolic capacity of the muscle, liver and other tissues in order to conserve the energy supply of vital organs (e.g. brain and heart) during hypoxic conditioning.

Taurine is an antioxidant sulfur-containing amino acid which is abundant in mammalian tissues. The results of the present study demonstrated that, compared with the $\mathrm{H} 0$ group, the serum content of taurine significantly increased in the mice in the H1 and HPC groups; although the increase in the HPC group was significant, the underlying mechanism was not clarified. This may be attributed to the impaired function 
Table II. Result from Metabolic Pathway Analysis with MetaboAnalyst3.0.

\begin{tabular}{|c|c|c|c|c|c|}
\hline Pathway name & Total cmpd & Hits & Raw P-value & $-\log (\mathrm{p})$ & Impact \\
\hline Phenylalanine, tyrosine and tryptophan biosynthesis & 4 & 2 & $1.23 \times 10^{-7}$ & 15.914 & 1.0000 \\
\hline Linoleic acid metabolism & 6 & 1 & $1.22 \times 10^{-6}$ & 13.621 & 1.0000 \\
\hline Valine, leucine and isoleucine biosynthesis & 11 & 2 & $1.46 \times 10^{-6}$ & 13.437 & 0.6667 \\
\hline Taurine and hypotaurine metabolism & 8 & 1 & $9.70 \times 10^{-9}$ & 18.451 & 0.4286 \\
\hline Phenylalanine metabolism & 11 & 2 & $1.23 \times 10^{-7}$ & 15.914 & 0.4074 \\
\hline Arachidonic acid metabolism & 36 & 2 & $2.76 \times 10^{-3}$ & 5.893 & 0.3260 \\
\hline Glyoxylate and dicarboxylate metabolism & 18 & 1 & $6.92 \times 10^{-8}$ & 16.487 & 0.2581 \\
\hline Tyrosine metabolism & 44 & 1 & $4.65 \times 10^{-8}$ & 16.883 & 0.1405 \\
\hline Citrate cycle & 20 & 2 & $2.06 \times 10^{-18}$ & 40.726 & 0.0792 \\
\hline
\end{tabular}

Total cmpd is the total number of compounds (cmpd) in the pathway; the hits is matched number from the user uploaded data; the raw P-value is the original P-value calculated from the enrichment analysis; the impact is the pathway impact value calculated from pathway topology analysis.

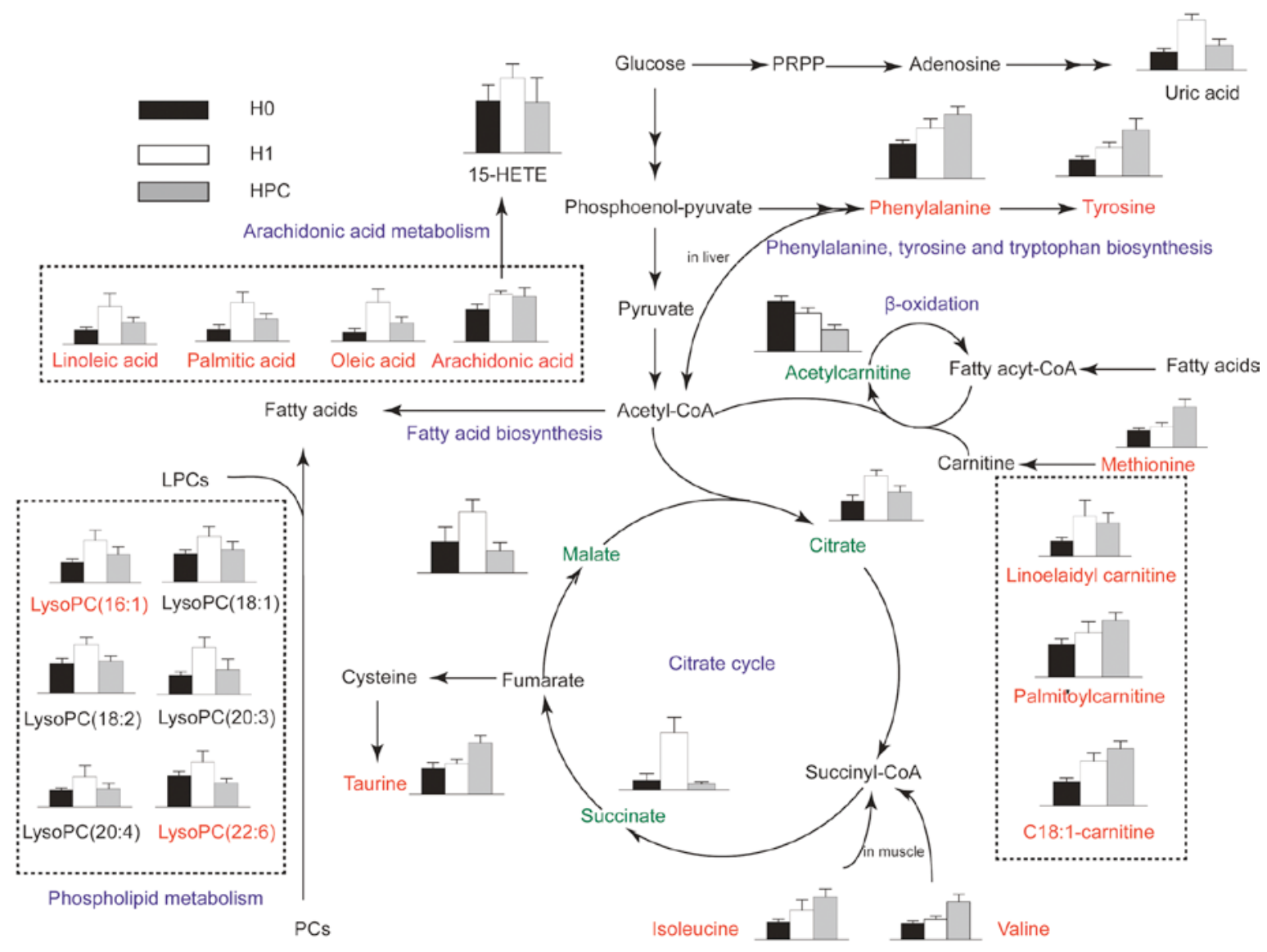

Figure 5. Schematic overview of the metabolites and major metabolic pathways. Red represents increased metabolites; green represents decreased metabolites; blue represents associated pathways; black indicates that these were not detected in the present study. H0, normoxic control; H1, acute hypoxic; HPC, acute repetitive hypoxia; PRPP, phosphoribosyl pyrophosphate; 15-HETE, 15-hydroxyeicosatetraenoic acid; acetyl-CoA, acetyl-coenzyme A; LysoPC, lysophosphatidylcholine.

of the mitochondrial membrane $\mathrm{Na}^{+} / \mathrm{K}^{+}$-ATP and the accumulation of intracellular $\mathrm{Na}^{+}$, leading to restricted mitochondrial oxidative phosphorylation, a decrease in ATP production, accumulation of intracellular lactic acid, and the activation of the cellular regulatory volume decrease reaction. Therefore, taurine with the reduction of other extracellular osmotic substances increased the content of taurine in the serum. Previous studies have demonstrated that taurine modulated 
neuronal activity, regulated the balance of intracellular and extracellular calcium ions, and protected neurons and transplanted hearts from ischemic and hypoxic injury, in addition to other functions $(26,27)$. Therefore, it was hypothesized that HPC protected the body against hypoxic injury partially by upregulating the level of taurine.

The carnitine system (including free carnitine and acylcarnitines) is essential for cell energy metabolism as a carrier of long-chain fatty acids for $\beta$-oxidation or as a reservoir pool of acyl-coenzyme A (28). In the present study, the difference in the abundance of carnitines, and their precursor methionine, among H0, H1 and HPC samples may indicate an association between the requirement of alternative energy and HPC. Previous studies have reported that acute hypoxia inhibited the free fatty acid (FFA) oxidation, which resulted in the accumulation of long-chain acylcarnitines $(19,29)$. In agreement with these previous reports, our results revealed three long-chain acylcarnitines (linoelaidylcarnitine, palmitoylcarnitine and octadecenoylcarnitine) that were significantly increased in the H1 and HPC groups, with the highest levels in the HPC group. In contrast with short-chain acylcarnitines, acetyl-L-carnitine was decreased in the H1 and HPC samples, with the lowest levels in the HPC group; however, the reason remains to be elucidated. Previous studies demonstrated that the administration of acetyl-L-carnitine attenuated neuronal damage, prevented apoptosis and improved energy status during hypoxic stress $(30,31)$. Therefore, it was hypothesized that supplementary short-chain carnitines may attenuate the damage of hypoxia by promoting $\beta$-oxidation of long-chain fatty acids, that decrease the accumulation of toxic long-chain acylcarnitines.

The results of the present study indicated that lysophosphatidylcholines (LysoPCs) [(LysoPC (16:1), LysoPC (18:2), LysoPC (18:1), LysoPC (20:4), LysoPC (20:3) and LysoPC (22:6)] and FFAs (arachidonic acid, linoleic acid, oleic acid, and palmitic acid) were significantly increased in the $\mathrm{H} 1$ group and decreased in the HPC group. These alterations may be putatively ascribed to the activation of phospholipase A2 (PLA2), which mediates the release of LysoPCs and specific fatty acids from PC. Reportedly, PLA2 was significantly increased under hypoxia, whereas it decreased gradually to a normal level with the formation of HPC (32); this result indirectly substantiated the results of the present study. LysoPCs are able to mediate a number of cell signaling pathways in monocytes/macrophages $(33,34)$ and specific receptors $(35)$, and therefore participate in the inflammatory response. The other product of PC metabolism, arachidonic acid, may be metabolized by cyclooxygenases and lipoxygenases forming various eicosanoids, including prostaglandins, thromboxanes, leukotrienes and lipoxins, that participate in the inflammatory response (36). In the present study, 15-hydroxyeicosatetraenoic acid, a product of arachidonic acid metabolisms, catalyzed by lipoxygenase, exhibited a trend similar to that of arachidonic acid. The results of the present study indicated that the phospholipid and arachidonic acid metabolic pathway may serve a principal role in HPC.

Uric acid is the end product of purine metabolism and is produced by two consecutive oxidation-reduction reactions catalyzed by xanthine oxidase $(\mathrm{XO})$ : Hypoxanthine to xanthine, and xanthine to uric acid. The results of the present study demonstrated that the level of uric acid in the H1 group was significantly increased compared with that in the $\mathrm{HO}$ and HPC groups. The mechanism may be associated with the reduction in ATP levels when increased adenine nucleotide turnover was coupled with the stimulation of XO (37).

The citrate cycle is central to aerobic metabolism, facilitating an adequate supply of substrates derived from carbohydrates, fatty acids or specific amino acids. In the present study, three pivotal intermediates of the citrate cycle (malate, citrate and succinate) were observed to be upregulated in the H1 group and downregulated in the HPC group. Therefore, mitochondrial aerobic respiration may have been inhibited by acute hypoxia, which may be partially restored by HPC.

In conclusion, a UPLC-QTOFMS-based serum metabolomic approach was developed to profile HPC-associated metabolic alterations. The results of the present study demonstrated that the HPC mouse model was well-established. In the present study, 25 significantly-altered metabolites were identified and the major metabolite was network predicted using pattern recognition and pathway analysis. The identified target metabolites were observed to encompass a variety of pathways associated with valine, leucine, and isoleucine biosynthesis, in addition to taurine, hypotaurine, phenylalanine, linoleic acid and arachidonic acid metabolism. The results of the present study provided novel insights into the protective mechanism of HPC.

\section{Acknowledgements}

The present study was supported by the National Natural Science Foundation of China (grant nos. 81301685 and J1310001), the 973 Project of China (grant no. 2012CB518201) and the Key Research Project of the People's Liberation Army (grant no. BWS11J042).

\section{References}

1. Janoff A: Alterations in lysosomes (intracellular enzymes) during shock; effects of preconditioning (tolerance) and protective drugs. Int Anesthesiol Clin 2: 251-269, 1964.

2. Dirnagl U, Becker K and Meisel A: Preconditioning and tolerance against cerebral ischaemia: From experimental strategies to clinical use. Lancet Neurol 8: 398-412, 2009.

3. Zhang SX, Miller JJ, Gozal D and Wang Y: Whole-body hypoxic preconditioning protects mice against acute hypoxia by improving lung function. J Appl Physiol (1985) 96: 392-397, 2004.

4. Carini R, De Cesaris MG, Splendore R, Bagnati M and Albano E: Ischemic preconditioning reduces $\mathrm{Na}(+)$ accumulation and cell killing in isolated rat hepatocytes exposed to hypoxia. Hepatology 31: 166-172, 2000.

5. Zhu HL, Wei X, Qu SL, Zhang C, Zuo XX, Feng YS, Luo Q, Chen GW, Liu MD, Jiang L, et al: Ischemic postconditioning protects cardiomyocytes against ischemia/reperfusion injury by inducing MIP2. Exp Mol Med 43: 437-445, 2011.

6. Gao Y, Gao G, Long C, Han S, Zu P, Fang L and Li J: Enhanced phosphorylation of cyclic AMP response element binding protein in the brain of mice following repetitive hypoxic exposure. Biochem Biophys Res Commun 340: 661-667, 2006.

7. Wang MM, Wang LJ, Chen Y, Li XR, Lv GW and Xue M: Establishment of the prediction model of tolerance time of mice and rats exposed to hypoxia. Chin J Comp Med: 21-23, 2008.

8. Lu GW, Yu S, Li RH, Cui XY and Gao CY: Hypoxic preconditioning: A novel intrinsic cytoprotective strategy. Mol Neurobiol 31: 255-271, 2005. 
9. Lv H, Wang Q, Wu S, Yang L, Ren P, Yang Y, Gao J and Li L: Neonatal hypoxic ischemic encephalopathy-related biomarkers in serum and cerebrospinal fluid. Clin Chim Acta 450: 282-297, 2015.

10. Hernández-Jiménez $\mathrm{M}$, Sacristán $\mathrm{S}$, Morales $\mathrm{C}$, García-Villanueva M, García-Fernández E, Alcázar A, González VM and Martín ME: Apoptosis-related proteins are potential markers of neonatal hypoxic-ischemic encephalopathy (HIE) injury. Neurosci Lett 558: 143-148, 2014.

11. Cui C, Zhou T, Li J, Wang H, Li X, Xiong J, Xu P and Xue M: Proteomic analysis of the mouse brain after repetitive exposure to hypoxia. Chem Biol Interact 236: 57-66, 2015.

12. Fiehn O: Metabolomics-the link between genotypes and phenotypes. Plant Mol Biol 48: 155-171, 2002.

13. Sreekumar A, Poisson LM, Rajendiran TM, Khan AP, Cao Q, Yu J, Laxman B, Mehra R, Lonigro RJ, Li Y, et al: Metabolomic profiles delineate potential role for sarcosine in prostate cancer progression. Nature 457: 910-914, 2009.

14. Jové M, Portero-Otín M, Naudí A, Ferrer I and Pamplona R Metabolomics of human brain aging and age-related neurodegenerative diseases. J Neuropathol Exp Neurol 73: 640-657, 2014

15. Shah SH, Kraus WE and Newgard CB: Metabolomic profiling for the identification of novel biomarkers and mechanisms related to common cardiovascular diseases: Form and function. Circulation 126: 1110-1120, 2012.

16. Armitage EG and Barbas C: Metabolomics in cancer biomarker discovery: Current trends and future perspectives. J Pharm Biomed Anal 87: 1-11, 2014.

17. Zhou T, Wang M, Cheng H, Cui C, Su S, Xu P and Xue M: UPLC-HRMS based metabolomics reveals the sphingolipids with long fatty chains and olefinic bonds up-regulated in metabolic pathway for hypoxia preconditioning. Chem Biol Interact 242: 145-152, 2015.

18. Lu GW, Chui XY and Zhao LF: Brain mechanisms of hypoxic preconditioning. Zhongguo Ying Yong Sheng Li Xue Za Zhi 20: 98-103, 2004 (In Chinese)

19. Liao WT, Liu B, Chen J, Cui JH, Gao YX, Liu FY, Xu G, Sun BD, Yuan ZB, Zhang EL, et al: Metabolite Modulation in Human Plasma in the Early Phase of Acclimatization to Hypobaric Hypoxia. Sci Rep 6: 22589, 2016.

20. Yin P, Wan D, Zhao C, Chen J, Zhao X, Wang W, Lu X, Yang S, Gu J and Xu G: A metabonomic study of hepatitis B-induced liver cirrhosis and hepatocellular carcinoma by using RP-LC and HILIC coupled with mass spectrometry. Mol BioSyst 5: 868-876, 2009.

21. Tan G, Liao W, Dong X, Yang G, Zhu Z, Li W, Chai Y and Lou Z: Metabonomic profiles delineate the effect of traditional Chinese medicine sini decoction on myocardial infarction in rats. PLoS One 7: e34157, 2012.

22. Xia J, Mandal R, Sinelnikov IV, Broadhurst D and Wishart DS MetaboAnalyst 2.0-a comprehensive server for metabolomic data analysis. Nucleic Acids Res 40: W127-W133, 2012.

23. Xia J and Wishart DS: Web-based inference of biological patterns, functions and pathways from metabolomic data using MetaboAnalyst. Nat Protoc 6: 743-760, 2011
24. Muratsubaki $\mathrm{H}$ and Yamaki A: Profile of plasma amino Acid levels in rats exposed to acute hypoxic hypoxia. Indian J Clin Biochem 26: 416-419, 2011

25. Walker V, Gentry AJ, Green LR, Hanson MA and Bennet L: Effects of hypoxia on plasma amino acids of fetal sheep. Amino Acids 18: 147-156, 2000.

26. Tang DQ, Bian TT, Zheng XX, Li Y, Wu XW, Li YJ, Du Q and Jiang SS: LC-MS/MS methods for the determination of edaravone and/or taurine in rat plasma and its application to a pharmacokinetic study. Biomed Chromatogr 28: 1173-1182, 2014.

27. Sahin MA, Yucel O, Guler A, Doganci S, Jahollari A, Cingoz F, Arslan S, Gamsizkan M, Yaman $\mathrm{H}$ and Demirkilic U: Is there any cardioprotective role of Taurine during cold ischemic period following global myocardial ischemia? J Cardiothorac Surg 6: $31,2011$.

28. Jones LL, McDonald DA and Borum PR: Acylcarnitines: Role in brain. Prog Lipid Res 49: 61-75, 2010.

29. Bruder ED and Raff H: Cardiac and plasma lipid profiles in response to acute hypoxia in neonatal and young adult rats. Lipids Health Dis 9: 3, 2010.

30. Hota KB, Hota SK, Chaurasia OP and Singh SB Acetyl-L-carnitine-mediated neuroprotection during hypoxia is attributed to ERK1/2-Nrf2-regulated mitochondrial biosynthesis. Hippocampus 22: 723-736, 2012.

31. Barhwal K, Singh SB, Hota SK, Jayalakshmi K and Ilavazhagan G: Acetyl-L-carnitine ameliorates hypobaric hypoxic impairment and spatial memory deficits in rats. Eur J Pharmacol 570: 97-107, 2007.

32. Liu H, Zhao H, Wang W, Xie X and Lu GW: Changes of PLA2 Activity in Hypoxic Preconditioning in Brain Tissue. Aca Periodical Changchun Coll Traditional Chinese Med 16: 49-50, 2006.

33. Duong CQ, Bared SM, Abu-Khader A, Buechler C, Schmitz A and Schmitz G: Expression of the lysophospholipid receptor family and investigation of lysophospholipid-mediated responses in human macrophages. Biochim Biophys Acta 1682: 112-119, 2004.

34. Kabarowski JH: G2A and LPC: Regulatory functions in immunity. Prostaglandins Other Lipid Mediat 89: 73-81, 2009.

35. Oestvang J and Johansen B: PhospholipaseA2: A key regulator of inflammatory signalling and a connector to fibrosis development in atherosclerosis. Biochim Biophys Acta 1761: 1309-1316, 2006.

36. Balsinde J, Winstead MV and Dennis EA: Phospholipase A(2) regulation of arachidonic acid mobilization. FEBS Lett 531: 2-6, 2002.

37. Hare JM and Johnson RJ: Uric acid predicts clinical outcomes in heart failure: Insights regarding the role of xanthine oxidase and uric acid in disease pathophysiology. Circulation 107: 1951-1953, 2003. 